\title{
Pretreatment Period
}

National Cancer Institute

\section{Source}

National Cancer Institute. Pretreatment Period. NCI Thesaurus. Code C103341.

The time prior to the treatment of interest. 